\title{
Second-order integro-differential parabolic variational inequalities arising from the valuation of American option
}

\author{
Yudong Sun ${ }^{*}$, Yimin Shi and Min Wu
}

\section{"Correspondence:}

yudongsun@yeah.net

Department of Applied

Mathematics, Northwestern

Polytechnical University, Xi'an,

710072, China

\begin{abstract}
This article deals with the existence and uniqueness of solution to the $\mathbb{R}_{d_{1}}$-valued parabolic variational inequalities with integro-differential terms which arise from the valuation of American option. The authors use the penalty method to construct a sequence of approximation parabolic problem and hence obtain the existence and uniqueness of solution to the approximation problem by using fixed point theory. Then the solution of parabolic variational inequalities is obtained by showing that the solution of this penalty problem converges to the variational inequalities. The uniqueness of the solution is also proven.
\end{abstract}

MSC: 35B40; 35K35

Keywords: American option; integro-differential; variational inequalities; existence; uniqueness

\section{Introduction}

In this paper, we are concerned with the existence and uniqueness of solution to an $\mathbb{R}_{d_{1}}$ valued parabolic variational inequality with the integro-differential terms

$$
\begin{cases}\left(u^{k}\right)_{t}-L_{k} u-F_{k}(x, t, u, \nabla u) \geq 0, & \text { in } Q_{T}, \\ u^{k}(x, t) \geq u_{0}^{k}(x), & \text { in } Q_{T}, \\ \left(\left(u^{k}\right)_{t}-L_{k} u-F_{k}(x, t, u, \nabla u)\right)\left(u^{k}-u_{0}^{k}(x)\right)=0, & \text { in } Q_{T}, \\ u^{k}(x, 0)=u_{0}^{k}(x), & \text { on } \Omega, \\ u^{k}(x, t)=g^{k}(x, t), & \text { on } \partial \Omega \times(0, T),\end{cases}
$$

where $\Omega \in \mathbb{R}_{d}$ is an open set with a smooth boundary $\partial \Omega, Q_{T}=\Omega \times[0, T]$ is a parabolic domain for some $T>0 . L=\left(L_{1}, L_{2}, \ldots, L_{d_{1}}\right)$, and $L_{k}=L_{k}(x, t)$ is a divergence form secondorder elliptic operator satisfying

$$
L_{k} u=\sum_{r=1}^{d_{1}} \sum_{i, j=1}^{d} \frac{\partial}{\partial x_{j}}\left(a_{k r}^{i j}(x, t) \frac{\partial}{\partial x_{i}} u^{r}\right)-\sum_{r=1}^{d_{1}} \sum_{i=1}^{d} b_{k r}^{i}(x, t) \frac{\partial}{\partial x_{i}} u^{r}-\sum_{r=1}^{d_{1}} c_{k r}(x, t) u^{r} .
$$

Moreover, the integro-differential operator $F_{k}$ is defined by

$$
F_{k}(x, t, u, \Delta u)=\int_{\Omega} f_{k}(x, t, y, u(x, t), \nabla u(x, t)) \mathrm{d} y
$$

which is a continuous integral operator as defined in [1].

\section{Springer}

(2014 Sun et al.; licensee Springer. This is an Open Access article distributed under the terms of the Creative Commons Attribution License (http://creativecommons.org/licenses/by/2.0), which permits unrestricted use, distribution, and reproduction in any medium, provided the original work is properly cited. 
The concrete motivations of studying (1) can be easily found in the literature. If $d_{1}=1$, $F_{k}(x, t, u, \Delta u)=0$, and the operator $L_{k}$ degenerates to

$$
L u=\partial_{t} u-\frac{\sigma^{2}}{2} \frac{\partial^{2}}{\partial x^{2}} u+\left(\frac{\sigma^{2}}{2}-r\right) \frac{\partial}{\partial x} u+r u,
$$

(1) becomes the linear variational inequality based on the famous Black-Scholes equation (see [2]). Such a variational inequality arises in many applications of American option pricing (see $[3,4])$. To deal with this problem, some scholars often introduce a free and moving boundary problem. By adding a certain penalty term to the Black-Scholes equation, the solution of this variational inequality is extended to a fixed domain. Furthermore, this penalty term forces the solution to stay above the payoff function at expiry. Throughout the last decade, a number of papers addressing penalty schemes for American options have been published (see, for instance, [5-9] and references therein).

However, several Black-Scholes models proposed in recent years, such as the model found in $[8,9]$, have allowed the risk assets driven by levy processes. In this model, the risk asset $S$ as in the Black-Scholes model, follows the stochastic process (assume $d_{1}=1$ )

$$
\mathrm{d} S_{t}=\mu S_{t} \mathrm{~d} t+\sigma S_{t} \mathrm{~d} z_{t}
$$

from which we obtain the following PIDE:

$$
\partial_{t} u=\frac{\sigma^{2}}{2}\left[\partial_{x x} u-\partial_{x} u\right]+\int\left[u(x+y, t)-u(x, t)-\left(e^{y}-1\right) \partial_{x} u\right] v(\mathrm{~d} x),
$$

where $z_{t}$ is a kind of levy process ( for details, see [10, 11]). The authors in [9] generalize (2) and prove the existence and uniqueness of a classical solution to a more general problem in the parabolic domain $Q_{T}=\Omega \times[0, T]$, where $\Omega$ is an open, unbounded subset of $\mathbb{R}_{d}$, with a smooth boundary $\partial \Omega$. A related work in the context of quantum mechanics has been studied in $[12,13]$.

Therefore, the authors of this paper intend to study a more complex variational inequality involved in American option based on the more complicated PIDE type Black-Scholes equation than (2). And we will consider $\mathbb{R}_{d_{1}}$-valued parabolic variational inequality (1) using the penalty method. The rest of this article is as follows. In Section 2, we state the main result. Section 3 discusses the penalty problem which will be used to prove our main result. In Section 4, we show the proof of our main result.

\section{Main result}

In this section, we present some notations and lemmas which are important to be used to prove our main result. Define $\mathbb{L}_{p}(\Omega)=L_{p}\left(\Omega ; \mathbb{R}_{d_{1}}\right)$ as the space of all $\mathbb{R}_{d_{1}}$-value functions $u=\left(u^{1}, u^{2}, \ldots, u^{d_{1}}\right)$ satisfying

$$
\|u\|_{\mathbb{L}_{p}(\Omega)}^{p}=\sum_{k=1}^{d_{1}}\left\|u^{k}\right\|_{L_{p}\left(\mathbb{R}_{d}\right)}^{p}<\infty
$$

Let $1 \leq p \leq \infty$ and $k \in \mathbb{N}$. By $W_{p}^{k}$, we mean that the $\mathbb{R}$-value functions space satisfies

$$
W_{p}^{k}(\Omega)=\left\{u \in L^{p}(\Omega ; \mathbb{R}) \mid D^{\alpha} u \in L^{p}(\Omega ; \mathbb{R}), 1 \leq \alpha \leq k\right\}
$$


which is Banach spaces with the norm

$$
\|v\|_{W_{p}^{k}(\Omega)}=\sum_{0 \leq|\alpha| \leq k}\left\|D^{\alpha} v\right\|_{L^{p}(\Omega)} .
$$

Further, we use the following Sobolev spaces which are modified from $\mathbb{R}$-value version to $\mathbb{R}_{d_{1}}$-value version

$$
\mathbb{W}_{p}^{k}(\Omega)=\left\{u \in L^{p}\left(\Omega ; \mathbb{R}_{d_{1}}\right) \mid D^{\alpha} u \in L^{p}\left(\Omega ; \mathbb{R}_{d_{1}}\right), 1 \leq \alpha \leq k\right\}
$$

with the norm

$$
\|v\|_{\mathbb{W}_{p}^{k}(\Omega)}=\sum_{0 \leq|\alpha| \leq k}\left\|D^{\alpha} v\right\|_{\mathbb{L}^{p}(\Omega)} .
$$

As mentioned in [14], we shall use $C^{2 k+\delta, k+\delta / 2}\left(\bar{Q}_{T}\right)$ to denote the Holder space which satisfies

$$
C^{2 k+\delta, k+\delta / 2}\left(\bar{Q}_{T}\right)=\left\{u\left|D^{\alpha} \partial_{t}^{\rho} \in C^{\delta, \delta / 2}\left(\bar{Q}_{T}\right),\right| \alpha \mid+2 \rho \leq 2 k\right\} .
$$

For $0<T<\infty$, we also use some following common notation from PDEs without mentioning:

$$
\begin{aligned}
& H^{k}(\Omega)=W_{2}^{k}(\Omega), \quad\|\cdot\|_{H^{k}(\Omega)}=\|\cdot\|_{W_{2}^{k}(\Omega)}, \\
& \mathbb{H}^{k}(\Omega)=\mathbb{W}_{2}^{k}(\Omega), \quad\|\cdot\|_{\mathbb{H}^{k}(\Omega)}=\|\cdot\|_{\mathbb{W}_{2}^{k}(\Omega)}, \\
& L^{p}\left(0, T ; H^{k}(\Omega)\right)=\left\{v(\cdot, t) \mid v(\cdot, t) \in H^{k}(\Omega) \text { in }(0, T) ;\|v(\cdot, t)\|_{H^{k}(\Omega)} \in L^{p}(0, T)\right\}, \\
& \mathbb{L}^{p}\left(0, T ; \mathbb{H}^{k}(\Omega)\right)=\left\{v(\cdot, t) \mid v(\cdot, t) \in \mathbb{H}^{k}(\Omega) \text { in }(0, T) ;\|v(\cdot, t)\|_{\mathbb{H}^{k}(\Omega)} \in L^{p}(0, T)\right\}, \\
& \|u\|_{L^{p}\left(0, T ; H^{k}(\Omega)\right)}^{p}=\int_{0}^{T}\|u(\cdot, t)\|_{H^{k}(\Omega)}^{p} \mathrm{~d} t, \quad\|u\|_{L^{p}\left(0, T ; \mathbb{H}^{k}(\Omega)\right)}^{p}=\int_{0}^{T}\|u(\cdot, t)\|_{\mathbb{H}^{k}(\Omega)}^{p} \mathrm{~d} t,
\end{aligned}
$$

where $0<T<\infty$.

Moreover, we use the following lemmas to show the existence and uniqueness of the solution for the penalty approximation of our main problem.

Definition 2.1 Assume that $X$ is a real Banach space, the space $C([0, T] ; X)$ consists of all continuous functions $u:[0, T] \rightarrow X$ with

$$
\|u\|_{C([0, T] ; X)}=\max _{0 \leq t \leq T}\|u\|_{X}<\infty
$$

Then $C([0, T] ; X)$ is a Banach space endowed with the norm $\|u\|_{C([0, T] ; X)}$.

Definition 2.2 A mapping $A: X \rightarrow X$ is called to be compact if and only if the sequence $\{A[u]\}_{k=1}^{\infty}$ is precompact for each bounded sequence $\{u\}_{k=1}^{\infty}$, that is, there exists a subsequence $\left\{u_{k_{j}}\right\}_{j=1}^{\infty}$ such that $\left\{A\left[u_{k_{j}}\right]\right\}_{j=1}^{\infty}$ converges in $X$. 
Lemma 2.3 (Schaefer's fixed point theorem) Suppose that $A: X \rightarrow X$ is a continuous and compact mapping. Further, assume that the set

$$
\{u \in X: u=\lambda A[u] \text {, for some } 0 \leq \lambda \leq 1\}
$$

is bounded. Then A has a fixed point.

The following two lemmas from the linear theory of parabolic partial differential equations can be found in [15].

Lemma 2.4 (Energy estimates) Consider the problem

$$
\begin{cases}\frac{\partial \nu^{k}}{\partial t}-L_{k} v=f_{k}(x, t), & \text { in } Q_{T}, \\ v^{k}(x, 0)=0, & \text { on } \Omega, \\ v^{k}(x, t)=0, & \text { on } \partial \Omega \times(0, T)\end{cases}
$$

with $f_{k} \in L^{2}\left(0, T ; L^{2}(\Omega)\right)$. Then there exists a unique solution $v \in L^{2}\left(0, T ; \mathcal{H}_{0}^{1}\left(B_{R}\right)\right) \cap$ $C\left([0, T] ; \mathcal{L}^{2}\left(B_{R}\right)\right)$ to problem $(9)$ that satisfies

$$
\max _{0<t<T}\|v(t)\|_{\mathbb{L}^{2}(\Omega)}+\|v(t)\|_{L^{2}\left(0, T ; \mathbb{H}_{0}^{1}(\Omega)\right)}+\left\|\frac{\partial v}{\partial t}\right\|_{L^{2}\left(0, T ; \mathbb{H}_{0}^{-1}(\Omega)\right)} \leq C\|f\|_{L^{2}\left(0, T ; \mathbb{L}^{2}(\Omega)\right)},
$$

where $C$ is a positive constant depending only on $\Omega, T$ and the operator $L$.

Lemma 2.5 (Improved regularity) There exists a unique weak solution

$$
u \in L^{2}\left(0, T ; \mathbb{H}_{0}^{1}\left(B_{R}\right)\right) \cap C\left([0, T] ; \mathbb{L}^{2}\left(B_{R}\right)\right)
$$

to problem (3), with $\frac{\partial u}{\partial t} \in L^{2}\left(0, T ; \mathbb{H}^{-1}(\Omega)\right)$. Moreover,

$$
u \in L^{2}\left(0, T ; \mathbb{H}^{2}(\Omega)\right) \cap L^{\infty}\left(0, T ; \mathbb{H}_{0}^{1}(\Omega)\right), \quad \frac{\partial u}{\partial t} \in L^{2}\left(0, T ; \mathbb{H}^{-1}(\Omega)\right)
$$

We also have the estimate

$$
\begin{aligned}
& \underset{0<t<T}{\operatorname{ess} \sup }\|u(t)\|_{\mathbb{H}_{0}^{1}(\Omega)}+\|u(t)\|_{L^{2}\left(0, T ; \mathbb{H}^{2}(\Omega)\right)}+\left\|\frac{\partial u}{\partial t}\right\|_{L^{2}\left(0, T ; \mathbb{L}^{2}(\Omega)\right)} \\
& \quad \leq C\|f\|_{L^{2}\left(0, T ; \mathbb{L}^{2}(\Omega)\right)},
\end{aligned}
$$

where $C$ is a positive constant depending only on $\Omega, T$ and the operator $L$.

Throughout this section, we impose the following assumptions:

(A1) The coefficients $a_{k r}^{i j}(x, t), b_{k r}^{i}(x, t), c_{k r}(x, t)$ belong to the Holder space $C^{\delta, \delta / 2}\left(\bar{Q}_{T}\right)$.

(A2) The operator $\frac{\partial}{\partial t}-L_{k}$ is (uniformly) parabolic, that is, there exists a constant $\theta>0$ such that

$$
\theta \sum_{k=1}^{d_{1}}\left|\nabla v^{k}\right|^{2} \leq \sum_{k, r=1}^{d_{1}} \sum_{i, j=1}^{d} a_{k r}^{i j}(x, t) \partial_{i} v^{r} \cdot \partial_{j} v^{k} \quad \text { for all }(x, t) \in Q_{T} v \in \mathbb{R}_{d}
$$


(A3) There exists a positive constant $B$ such that for all $v \in H_{0}^{1}(\Omega)$ and $(x, t) \in Q_{T}$, we have

$$
B \sum_{k=1}^{d_{1}} \partial_{i} v^{k} \cdot v^{k} \leq \sum_{k, r=1}^{d_{1}} b_{k r}^{i}(x, t) \partial_{i} v^{r} \cdot v^{k}, \quad c_{k r}(x, t)>0, k, r=1,2, \ldots, d_{1} .
$$

(A4) $u_{0}^{k}(x)$ and $g_{k}(x, t)$ belong to the Holder spaces $C^{2+\delta}\left(R^{d}\right)$ and $C^{2+\delta, 1+\delta / 2}\left(\bar{Q}_{T}\right)$, respectively. Moreover, for all $(x, t) \in Q_{T}$, we have that $u_{0}^{k}(x)<g_{k}(x, t), k=1,2, \ldots, d_{1}$.

(A5) The following two consistency conditions

$$
g_{k}(x, 0)=u_{0}^{k}(x) \text { and } g_{k}(x, 0)-L_{k}(x, 0) u_{0}^{k}(x)=0
$$

hold for all $x \in \partial \Omega, k=1,2, \ldots, d_{1}$.

(A6) $f_{k}(x, t, y, z, p)$ is nonnegative and belongs to $C^{1}\left(Q_{T} \times \Omega \times \mathbb{R}_{d_{1}} \times \mathbb{R}_{d_{1}}\right), k=1,2, \ldots, d_{1}$, and there exists $0<\varepsilon<1$ satisfying

$$
\left|f_{k}\left(\cdot, \cdot, \cdot, z_{1}, p_{1}\right)-f_{k}\left(\cdot, \cdot, \cdot, z_{2}, p_{2}\right)\right| \leq \varepsilon\left|z_{1}-z_{2}\right|+\varepsilon\left|p_{1}-p_{2}\right| .
$$

(A7) For some $C_{0}>0, f=\left(f_{1}, f_{2}, \ldots, f_{d_{1}}\right)$ satisfies the estimate

$$
\left|f_{k}(x, t, y, z, p)\right| \leq C_{0}\left(f_{0, k}+\sum_{r=1}^{d_{1}}\left|z^{r}\right|+\sum_{r=1}^{d_{1}}\left|p^{r}\right|\right)
$$

for all $(x, t, y, z, p) \in Q_{T} \times \Omega \times R_{d_{1}} \times R_{d_{1}}$, where $C_{0}$ is a positive constant independent of $f, f_{0, k} \in \mathbb{L}^{2}(\Omega)$.

(A8) If $u \in \mathbb{H}_{\text {loc }}^{2}\left(\mathbb{R}_{d}\right)$, then $F(x, t, u, \nabla u) \in L^{2}\left(0, T ; \mathbb{L}_{\text {loc }}^{2}(R)\right)$. If $w_{n} \rightarrow w$ in $L^{2}\left(0, T ; \mathbb{H}_{0}^{1}(\Omega)\right)$, then $F_{k}\left(x, t, w_{n}, \Delta w_{n}\right) \rightarrow F_{k}(x, t, w, \Delta w)$ in $L^{2}\left(0, T ; \mathbb{L}^{2}(\Omega)\right)$.

Using these assumptions, we will elaborate our main result.

Theorem 2.6 Under hypotheses (A1)-(A8), there exists a unique solution $u \in L^{2}(0, T$; $\left.\mathbb{H}^{2}(\Omega)\right) \cap C\left([0, T] ; \mathcal{L}^{2}(\Omega)\right)$ to variational inequality (1) satisfying

$$
\|u\|_{L^{2}\left(0, T ; \mathbb{H}_{0}^{2}(\Omega)\right)} \leq N\left(\left\|u_{0}\right\|_{\mathbb{L}^{2}(\Omega)}+\|g\|_{L^{2}\left(0, T ; \mathbb{L}^{2}(\Omega)\right)}+\|F(\cdot, \cdot, 0,0)\|_{L^{2}\left(0, T ; \mathbb{L}^{2}(\Omega)\right)}\right) .
$$

The proof of Theorem 2.6 will be given in Section 4 .

\section{The penalty problem}

In order to prove the existence and uniqueness of the solution, we consider the following penalty approximation of problem (1)

$$
\begin{cases}\left(u_{\varepsilon}^{k}\right)_{t}-L_{k} u_{\varepsilon}-F_{k}\left(x, t, u_{\varepsilon}, \nabla u_{\varepsilon}\right)+\beta_{\varepsilon}\left(u_{\varepsilon}^{k}-u_{0}^{k}(x)\right)=0, & \text { in } Q_{T}, \\ u_{\varepsilon}^{k}(x, 0)=u_{0}^{k}(x), & \text { on } \Omega, \\ u_{\varepsilon}^{k}(x, t)=g^{k}(x, t), & \text { on } \partial \Omega \times(0, T),\end{cases}
$$


where $\beta_{\varepsilon, k}(\cdot)$ is the penalty function satisfying

$$
\begin{aligned}
& 0<\varepsilon \leq 1, \quad \beta_{\varepsilon, k}(x) \in C^{2}(R), \quad \beta_{\varepsilon, k}(x) \leq 0, \quad \beta_{\varepsilon, k}^{\prime}(x) \geq 0, \quad \beta_{\varepsilon, k}^{\prime \prime}(x) \leq 0, \\
& \lim _{\varepsilon \rightarrow 0} \beta_{\varepsilon, k}(x)= \begin{cases}0, & x>0, \\
-\infty, & x<0 .\end{cases}
\end{aligned}
$$

Next, we introduce a change of variables

$$
v(x, t)=u_{\varepsilon}(x, t)-\varphi(x, t)
$$

to transform problem (5) into the zero boundary condition of the form

$$
\begin{cases}\left(v^{k}\right)_{t}-L_{k} v=F_{k}(x, t, v+\varphi, \nabla(v+\varphi))-\beta_{\varepsilon}\left(v^{k}+\varphi^{k}-u_{0}^{k}(x)\right), & \text { in } Q_{T}, \\ v^{k}(x, 0)=0, & \text { on } \Omega, \\ v^{k}(x, t)=\varphi^{k}(x, t)=0, & \text { on } \partial \Omega \times(0, T) .\end{cases}
$$

Here $\varphi=\left(\varphi^{1}, \varphi^{2}, \ldots, \varphi^{d_{1}}\right) \in C^{2+\delta, 1+\delta / 2}\left(\bar{Q}_{T}\right)$ is the unique solution to the problem

$$
\begin{cases}\left(u^{k}\right)_{t}-L_{k} u=0, & \text { in } Q_{T}, \\ u^{k}(x, 0)=u_{0}^{k}(x), & \text { on } \Omega, \\ u^{k}(x, t)=g_{k}(x, t), & \text { on } \partial \Omega \times(0, T)\end{cases}
$$

satisfying

$$
\|\varphi\|_{L^{2}\left(0, T ; \mathbb{H}^{2}(\Omega)\right)} \leq N\left(\left\|u_{0}\right\|_{\mathbb{L}^{2}(\Omega)}+\|g\|_{L^{2}\left(0, T ; \mathbb{L}^{2}(\Omega)\right)}\right) .
$$

For further details, see Theorem 10.4.1 in [15].

Definition 3.1 $v$ is said to be a weak solution to problem (7) if

$$
\nu \in L^{2}\left(0, T ; \mathbb{H}_{0}^{1}(\Omega)\right), \quad \frac{\partial v}{\partial t} \in L^{2}\left(0, T ; \mathbb{H}^{-1}(\Omega)\right)
$$

and

$$
\begin{aligned}
\int_{\Omega}\left(\frac{\partial v^{k}}{\partial t} g+\sum_{r=1}^{d_{1}} \sum_{i, j=1}^{d} a_{k r}^{i j}(x, t) \partial_{i} v^{r} \cdot \partial_{j} g\right) \mathrm{d} x \\
\quad+\int_{\Omega}\left(\sum_{r=1}^{d_{1}} \sum_{i=1}^{d} b_{k r}^{i}(x, t) \partial_{i} v^{r} \cdot g+\sum_{r=1}^{d_{1}} c_{k r}(x, t) v^{r} \cdot g\right) \mathrm{d} x \\
=\int_{\Omega} F_{k}(x, t, v+\varphi, \nabla(v+\varphi)) g \mathrm{~d} x-\int_{\Omega} \beta_{\varepsilon}\left(v^{k}+\varphi^{k}-u_{0}^{k}\right) g \mathrm{~d} x
\end{aligned}
$$

for all $g \in \mathbb{H}_{0}^{1}(\Omega)$.

Lemma 3.2 If $v \in L^{2}\left(0, T ; \mathbb{H}_{0}^{1}(\Omega)\right)$ and $\frac{\partial v}{\partial t} \in L^{2}\left(0, T ; \mathbb{H}^{-1}(\Omega)\right)$, then

$$
v \in C\left([0, T] ; \mathbb{L}^{2}(\Omega)\right)
$$


The mapping $t \rightarrow\|v(t)\|_{\mathbb{L}^{2}(\Omega)}^{2}$ is absolutely continuous with

$$
\frac{\mathrm{d}}{\mathrm{d} t}\left\|v^{k}(t)\right\|_{L^{2}(\Omega)}^{2}=2 \int_{\Omega} \frac{\partial v^{k}}{\partial t} v^{k} \mathrm{~d} t, \quad \frac{\mathrm{d}}{\mathrm{d} t}\|v(t)\|_{\mathbb{L}^{2}(\Omega)}^{2}=2 \sum_{k=1}^{d_{1}} \int_{\Omega} \frac{\partial v^{k}}{\partial t} \cdot v^{k} \mathrm{~d} t
$$

for any $0 \leq t \leq T$. The proof is quite standard and can be found in [15].

Lemma 3.3 If $v$ is a weak solution to problem (7), then there exists a positive constant $C$ such that

$$
\|v(t)\|_{L^{2}\left(0, T ; \mathcal{H}_{0}^{1}(\Omega)\right)} \leq C
$$

where $C$ is independent of $v$.

Proof Choosing $v \in \mathbb{H}_{0}^{1}(\Omega)$ as the test function in (9), we obtain

$$
\begin{aligned}
\int_{\Omega}\left(\frac{\partial v^{k}}{\partial t} v^{k}+\sum_{r=1}^{d_{1}} \sum_{i, j=1}^{d} a_{k r}^{i j}(x, t) \partial_{i} v^{r} \cdot \partial_{j} v^{k}\right) \mathrm{d} x \\
\quad+\int_{\Omega}\left(\sum_{r=1}^{d_{1}} \sum_{i, j=1}^{d} b_{k r}^{i}(x, t) \partial_{i} v^{r} \cdot v^{k}+\sum_{r=1}^{d_{1}} c_{k r}(x, t) v^{r} v^{k}\right) \mathrm{d} x \\
=\int_{\Omega} F_{k}(x, t, v+\varphi, \nabla(v+\varphi)) v \mathrm{~d} x-\int_{\Omega} \beta_{\varepsilon}\left(v^{k}+\varphi^{k}-u_{0}^{k}\right) v^{k} \mathrm{~d} x .
\end{aligned}
$$

From (1), we easily have that

$$
v^{k}+\varphi^{k} \geq u_{0}^{k} \quad \text { for all }(x, t) \in Q_{T} .
$$

This and (6) lead to

$$
\left|\int_{\Omega} \beta_{\varepsilon}\left(v^{k}+\varphi^{k}-u_{0}^{k}\right) v^{k} \mathrm{~d} x\right| \leq\left|\beta_{\varepsilon}(0)\right| \cdot \int_{\Omega}\left|v^{k}\right| \mathrm{d} x .
$$

By (A7), so that we get

$$
\begin{aligned}
& \left|F_{k}(x, t, v+\varphi, \nabla(v+\varphi))\right| \\
& \quad \leq\left|\int_{\Omega} f_{k}(x, t, y, v+\varphi, \nabla(v+\varphi)) \mathrm{d} y\right| \\
& \quad \leq C_{0} \int_{\Omega}\left(f_{0, k}+\sum_{k=1}^{d_{1}}\left|v^{k}\right|+\sum_{k=1}^{d_{1}}\left|\nabla v^{k}\right|+\sum_{k=1}^{d_{1}}\left|\varphi^{k}\right|+\sum_{k=1}^{d_{1}}\left|\nabla \varphi^{k}\right|\right) \mathrm{d} x,
\end{aligned}
$$

where $C_{0}$ is a positive constant which depends only on the region $\Omega$. It follows by (A2) and (A3) that

$$
\theta \sum_{k=1}^{d_{1}}\left|\nabla v^{k}\right|^{2} \leq \sum_{k, r=1}^{d_{1}} \sum_{i, j=1}^{d} a_{k r}^{i j} \partial_{i} v^{r} \cdot \partial_{j} v^{k}
$$


Sun et al. Journal of Inequalities and Applications 2014, 2014:8

Page 8 of 14

http://www.journalofinequalitiesandapplications.com/content/2014/1/8

$$
\begin{aligned}
\int_{\Omega} \sum_{k, r=1}^{d_{1}} \sum_{i=1}^{d} b_{k r}^{i}(x, t) \partial_{i} v^{r} \cdot v^{k} \mathrm{~d} x & \geq B \sum_{k=1}^{d_{1}} \sum_{i=1}^{d} \int_{\Omega} \partial_{i} v^{k} \cdot v^{k} \mathrm{~d} x \\
& =\frac{B}{2} \sum_{k=1}^{d_{1}} \sum_{i=1}^{d} \int_{\Omega} \partial_{i}\left(v^{k}\right)^{2} \mathrm{~d} x=0
\end{aligned}
$$

and

$$
\int_{\Omega} c_{k r}(x, t) v^{2} \mathrm{~d} x \geq 0
$$

Now, we use Theorem 10.4.1 in [15] and substitute (11)-(15) into (10) to arrive at

$$
\begin{aligned}
& \frac{1}{2} \frac{\mathrm{d}}{\mathrm{d} t}\left\|v^{k}\right\|_{\mathcal{L}^{2}(\Omega)}^{2}+\theta \sum_{k=1}^{d_{1}} \int_{\Omega}\left|\nabla v^{k}\right|^{2} \mathrm{~d} x \\
& \leq C_{0} \int_{\Omega}\left(f_{0, k}+\sum_{k=1}^{d_{1}}\left|v^{k}\right|+\sum_{k=1}^{d_{1}}\left|\nabla v^{k}\right|+\sum_{k=1}^{d_{1}}\left|\varphi^{k}\right|+\sum_{k=1}^{d_{1}}\left|\nabla \varphi^{k}\right|\right) \cdot \sum_{k=1}^{d_{1}}\left|v^{k}\right| \mathrm{d} x \\
& \quad+\left|\beta_{\varepsilon}(0)\right| \cdot \int_{\Omega} \sum_{k=1}^{d_{1}}\left|v^{k}\right| \mathrm{d} x .
\end{aligned}
$$

It follows, by using the Cauchy inequality with $\omega>0$, that

$$
\begin{aligned}
\frac{1}{2} \frac{\mathrm{d}}{\mathrm{d} t}\left\|v^{k}\right\|_{\mathbb{L}^{2}(\Omega)}^{2}+\theta \sum_{k=1}^{d_{1}} \int_{\Omega}\left|\nabla v^{k}\right|^{2} \mathrm{~d} x \\
\leq C_{0}\left(\omega \int_{\Omega} f_{0, k}^{2} \mathrm{~d} x+\frac{1}{4 \omega} \int_{\Omega}\left(\sum_{k=1}^{d_{1}}\left|v^{k}\right|\right)^{2} \mathrm{~d} x\right)+C_{0} \int_{\Omega}\left(\sum_{k=1}^{d_{1}}\left|v^{k}\right|\right)^{2} \mathrm{~d} x \\
+C_{0}\left(\omega \int_{\Omega}\left(\sum_{k=1}^{d_{1}}\left|\nabla v^{k}\right|\right)^{2} \mathrm{~d} x+\frac{1}{4 \omega} \int_{\Omega}\left(\sum_{k=1}^{d_{1}}\left|v^{k}\right|\right)^{2} \mathrm{~d} x\right) \\
+C_{0}\left(\omega \int_{\Omega}\left(\sum_{k=1}^{d_{1}}\left|\varphi^{k}\right|\right)^{2} \mathrm{~d} x+\frac{1}{4 \omega} \int_{\Omega}\left(\sum_{k=1}^{d_{1}}\left|v^{k}\right|\right)^{2} \mathrm{~d} x\right) \\
+C_{0}\left(\omega \int_{\Omega}\left(\sum_{k=1}^{d_{1}}\left|\nabla \varphi^{k}\right|\right)^{2} \mathrm{~d} x+\frac{1}{4 \omega} \int_{\Omega}\left(\sum_{k=1}^{d_{1}}\left|v^{k}\right|\right)^{2} \mathrm{~d} x\right) \\
+\left|\beta_{\varepsilon}(0)\right| \cdot\left(\omega \int_{\Omega} \mathrm{d} x+\frac{1}{4 \omega} \int_{\Omega}\left(\sum_{k=1}^{d_{1}}\left|v^{k}\right|\right)^{2} \mathrm{~d} x\right) .
\end{aligned}
$$

Next, we choose $0<\omega \ll 1$ to arrive at

$$
\frac{1}{2} \frac{\mathrm{d}}{\mathrm{d} t}\left\|v^{k}\right\|_{\mathbb{L}^{2}(\Omega)}^{2}+C_{1}\|v\|_{\mathbb{H}_{0}^{1}(\Omega)}^{2} \leq C_{2}\|v\|_{\mathbb{L}^{2}(\Omega)}^{2}+\tilde{C}(t),
$$

where $C_{1}$ and $C_{2}$ are positive constants and

$$
\tilde{C}(t)=C_{0} \omega \int_{\Omega}\left(f_{0, k}^{2}+\left(\sum_{k=1}^{d_{1}}\left|\varphi^{k}\right|\right)^{2}+\left(\sum_{k=1}^{d_{1}}\left|\nabla \varphi^{k}\right|\right)^{2}\right) \mathrm{d} x+\omega\left|\beta_{\varepsilon}(0)\right| \cdot \int_{\Omega} 1 \mathrm{~d} x .
$$


Note that $\varphi_{k} \in C^{2+\delta, 1+\delta / 2}\left(\bar{Q}_{T}\right)$. Therefore we choose a positive constant $C_{3}=\sup _{0<t<T} \tilde{C}(t)$ to obtain

$$
\frac{1}{2} \frac{d}{d t}\|v(t)\|_{\mathbb{L}^{2}(\Omega)}^{2}+C_{1}\|v\|_{\mathbb{H}_{0}^{1}(\Omega)}^{2} \leq C_{2}\|v\|_{\mathbb{L}^{2}(\Omega)}^{2}+C_{3} .
$$

On the one hand, letting $\eta(t)=\|v(t)\|_{\mathbb{L}^{2}(\Omega)}^{2},(17)$ gives

$$
\eta^{\prime}(t) \leq 2 C_{2} \eta(t)+2 C_{3} .
$$

Using the differential form of Gronwall inequality with $\eta(0)=\|v(0)\|_{\mathcal{L}^{2}(\Omega)}^{2}=0$, we get

$$
\|v(t)\|_{\mathbb{L}^{2}(\Omega)}^{2} \leq 2 e^{2 C_{2} t} C_{3} t
$$

and

$$
\max _{0<t<T}\|v(t)\|_{\mathbb{L}^{2}(\Omega)}^{2} \leq C_{4},
$$

where $C_{4}$ is a positive constant independent of $v$.

On the other hand, an integration of (17) from 0 to $T$ with $\|v(0)\|_{\mathbb{L}^{2}(\Omega)}^{2}=0$ yields

$$
C_{1} \int_{0}^{T}\|v\|_{\mathbb{H}_{0}^{1}(\Omega)}^{2} \mathrm{~d} t \leq C_{2} \int_{0}^{T}\|v\|_{\mathbb{L}^{2}(\Omega)}^{2} \mathrm{~d} t+C_{3} T+\lim _{t \rightarrow T^{-}}\|v(t)\|_{\mathbb{L}^{2}(\Omega)}^{2}
$$

Using inequality (18), we obtain

$$
\|v(t)\|_{L^{2}\left(0, T ; \mathbb{H}_{0}^{1}(\Omega)\right)} \leq \frac{C_{2} C_{4}}{C_{1}} T+\frac{C_{3}}{C_{1}} T+\frac{C_{4}}{C_{1}} .
$$

Thus, the proof is ended by letting $C=\frac{C_{2} C_{4}}{C_{1}} T+\frac{C_{3}}{C_{1}} T+\frac{C_{4}}{C_{1}}>0$.

Lemma 3.4 The solution to problem (7) satisfies

$$
u^{k}(x, t) \geq u_{0}^{k}(x)
$$

Proof Using (A8), there exists a positive constant $M$ satisfying

$$
\left|F_{k}(x, t, u, \nabla u)\right| \leq M \text {. }
$$

Here we plan to finish the proof by using contradiction. Assume that $\bigcup_{k=1}^{d_{1}}\left\{u^{k}(x, t)<u_{0}^{k}(x)\right\}$ is not empty, that is, at least there exists one $k$ satisfying $u^{k}<u_{0}^{k}$. Let $\beta_{\varepsilon}(0)=-M$, from (16), one gets

$$
u_{t}-L u=F_{k}(x, t, u, \nabla u)-\beta_{\varepsilon}\left(u^{k}-u_{0}^{k}(x)\right)>F_{k}(x, t, u, \nabla u)-\beta_{\varepsilon}(0) \geq 0 .
$$

Using the standard maximum principle and (A4), one gets

$$
u^{k}(x, t) \geq u_{0}^{k}(x)
$$


This is obviously contradictory. Therefore, we conclude that $u^{k}(x, t) \geq u_{0}^{k}(x)$ for any $(x, t) \in Q_{T}$.

Lemma 3.5 If (A1)-(A8) are satisfied, there exists a unique weak solution $v \in L^{2}(0, T$; $\left.\mathbb{H}_{0}^{2}(\Omega)\right) \cap C\left([0, T] ; \mathbb{L}^{2}(\Omega)\right)$ to problem (7) satisfying

$$
\|v\|_{L^{2}\left(0, T ; \mathbb{H}_{0}^{2}(\Omega)\right)} \leq N\|F(\cdot, \cdot, 0,0)\|_{L^{2}\left(0, T ; \mathbb{L}^{2}(\Omega)\right)}+N\left|\beta_{\varepsilon}(0)\right| T,
$$

where $N$ is a positive constant independent of $v$.

Proof Given $w \in L^{2}\left(0, T ; \mathbb{H}_{0}^{1}(\Omega)\right)$, set

$$
f_{w}=\left(f_{1, w}, f_{2, w}, \ldots, f_{d_{1}, w}\right), \quad f_{k, w}(x, t)=F_{k}(x, t, w+\varphi, \nabla(w+\varphi))-\beta_{\varepsilon}\left(w^{k}+\varphi^{k}-u_{0}^{k}\right) .
$$

The use of (A8) leads to

$$
f_{w}(x, t) \in L^{2}\left(0, T ; \mathbb{L}^{2}(\Omega)\right) .
$$

By Lemma 2.4, there exists a unique solution $v \in L^{2}\left(0, T ; \mathbb{H}_{0}^{2}(\Omega)\right) \cap C\left([0, T] ; \mathbb{L}^{2}(\Omega)\right)$ to

$$
\begin{cases}\left(v^{k}\right)_{t}-L_{k} v=f_{k, w}(x, t) & \text { in } Q_{T}, \\ v^{k}(x, 0)=0, & \text { on } \Omega, \\ v^{k}(x, t)=0, & \text { on } \partial \Omega \times(0, T) .\end{cases}
$$

Define the mapping

$$
M: L^{2}\left(0, T ; \mathbb{H}_{0}^{2}(\Omega)\right) \rightarrow L^{2}\left(0, T ; \mathbb{H}_{0}^{2}(\Omega)\right), \quad w \mapsto M(w)=v,
$$

where $v$ is derived from $w$ via (21).

Here we plan to prove the existence and uniqueness by Schaefer's fixed point theorem. So that we need to present the continuity and compactness of the mapping $M$. In this proof we only prove the continuity of the mapping $M$. The compactness can be obtained by following similar arguments, so we omit it here.

Let $\left\{w_{n}\right\}_{n} \in L^{2}\left(0, T ; \mathbb{H}_{0}^{1}(\Omega)\right)$ be a sequence such that

$$
w_{k} \rightarrow w \quad \text { in } L^{2}\left(0, T ; \mathbb{H}_{0}^{1}(\Omega)\right)
$$

By the improved regularity (4), we obtain

$$
\left\|v_{n}\right\|_{L^{2}\left(0, T ; \mathbb{H}^{2}(\Omega)\right)} \leq\left\|f_{w_{k}}\right\|_{L^{2}\left(0, T ; \mathbb{L}^{2}(\Omega)\right)} \quad \text { for } v_{n}=M\left[w_{n}\right], n=1,2, \ldots
$$

By using (A8) and Lemma 3.4 with $\beta_{\varepsilon}(x) \leq 0, \beta_{\varepsilon}^{\prime}(x) \geq 0$, we have

$$
\begin{aligned}
& \left\|f_{w_{k}}\right\|_{L^{2}\left(0, T ; \mathbb{L}^{2}(\Omega)\right)} \\
& \quad \leq\left\|F_{k}(x, t, w+\varphi, \nabla w+\nabla \varphi)\right\|_{L^{2}\left(0, T ; \mathbb{L}^{2}(\Omega)\right)}+\left|\beta_{\varepsilon}(0)\right| d_{1} T \\
& \quad \leq\left\|F_{k}(x, t, w+\varphi, \nabla w+\nabla \varphi)-F_{k}(x, t, 0,0)\right\|_{L^{2}\left(0, T ; \mathbb{L}^{2}(\Omega)\right)}
\end{aligned}
$$




$$
\begin{aligned}
& +\left\|F_{k}(x, t, 0,0)\right\|_{L^{2}\left(0, T ; \mathbb{L}^{2}(\Omega)\right)}+\left|\beta_{\varepsilon}(0)\right| d_{1} T \\
\leq & \alpha\|w\|_{L^{2}\left(0, T ; \mathbb{L}^{2}(\Omega)\right)}+\alpha\|\nabla w\|_{L^{2}\left(0, T ; \mathbb{L}^{2}(\Omega)\right)} \\
& +\left\|F_{k}(x, t, 0,0)\right\|_{L^{2}\left(0, T ; \mathbb{L}^{2}(\Omega)\right)}+\left|\beta_{\varepsilon}(0)\right| d_{1} T \\
\leq & \alpha\|w\|_{L^{2}\left(0, T ; \mathbb{H}^{2}(\Omega)\right)}+\left\|F_{k}(x, t, 0,0)\right\|_{L^{2}\left(0, T ; \mathbb{L}^{2}(\Omega)\right)}+\left|\beta_{\varepsilon}(0)\right| d_{1} T .
\end{aligned}
$$

The two inequalities above lead to

$$
\begin{aligned}
& \left\|v_{n}\right\|_{L^{2}\left(0, T ; \mathbb{H}^{2}(\Omega)\right)} \\
& \quad \leq \alpha\left\|w_{n}\right\|_{L^{2}\left(0, T ; \mathbb{H}^{2}(\Omega)\right)}+\left\|F_{k}(x, t, 0,0)\right\|_{L^{2}\left(0, T ; \mathbb{L}^{2}(\Omega)\right)}+\left|\beta_{\varepsilon}(0)\right| d_{1} T .
\end{aligned}
$$

Next, we pay our attention to the sequence $\left\{\left\|f_{w_{n}}\right\|_{L^{2}\left(0, T ; \mathbb{L}^{2}(\Omega)\right)}\right\}_{n}$. Since $w_{n} \rightarrow w$ in $L^{2}(0, T$; $\mathbb{H}_{0}^{1}(\Omega)$ ), from (A8) we have

$$
\left\|f_{w_{n}}(x, t)\right\|_{L^{2}\left(0, T ; \mathbb{L}^{2}(\Omega)\right)} \rightarrow\left\|f_{w}(x, t)\right\|_{L^{2}\left(0, T ; \mathbb{L}^{2}(\Omega)\right)} .
$$

This and (20) lead to the fact that the sequence $\left\{\left\|f_{w_{n}}\right\|_{L^{2}\left(0, T ; \mathbb{L}^{2}(\Omega)\right)}\right\}_{n}$ is bounded, that is,

$$
\left\|f_{w_{n}}\right\|_{L^{2}\left(0, T ; \mathbb{L}^{2}(\Omega)\right)} \leq C_{6} .
$$

Combing (23) with (24), the sequence $\left\{v_{n}\right\}_{n}$ is bounded uniformly in $L^{2}\left(0, T ; \mathbb{H}^{2}(\Omega)\right.$ ). In a similar way, $\left\{\frac{\partial v_{n}}{\partial t}\right\}_{n}$ is uniformly bounded in $L^{2}\left(0, T ; \mathbb{H}^{-1}(\Omega)\right)$. By using Rellich's theorem (see [16]), there exist a subsequence $\left\{v_{j}\right\}_{j} \in L^{2}\left(0, T ; \mathbb{H}_{0}^{1}(\Omega)\right)$ and a function $v \in$ $L^{2}\left(0, T ; \mathbb{H}_{0}^{1}(\Omega)\right)$ which satisfies

$$
v_{n_{j}} \rightarrow v \quad \text { in } L^{2}\left(0, T ; \mathbb{H}_{0}^{1}(\Omega)\right) \text { as } j \rightarrow \infty
$$

such that

$$
\int_{\Omega}\left(\frac{\partial v_{n_{j}}^{k}}{\partial t} \phi-L_{k} v_{n_{j}} \phi\right) \mathrm{d} x=\int_{\Omega} f_{k, w_{n_{j}}}(x, t) \phi \mathrm{d} x \quad \text { for each } \phi \in H_{0}^{1}(\Omega) .
$$

We combine (22) with (25) to arrive at

$$
\int_{\Omega}\left(\frac{\partial v^{k}}{\partial t} \phi-L_{k} v \phi\right) \mathrm{d} x=\int_{\Omega} f_{k, w}(x, t) \phi \mathrm{d} x
$$

Therefore,

$$
v=A[w], \quad A\left[w_{n}\right] \rightarrow A[w] \quad \text { in } L^{2}\left(0, T ; \mathbb{H}_{0}^{1}(\Omega)\right) .
$$

Further, by Lemma 3.3, we have that $\left\{w \in L^{2}\left(0, T ; \mathbb{H}_{0}^{1}(\Omega)\right): w=A[w]\right\}$ is bounded. Hence the existence and uniqueness of this theorem are proven by using Lemma 2.3 with $\lambda=1$.

Finally, we pay our attention to the estimate (19). Letting $n \rightarrow \infty$ in (23), we obtain

$$
\|v\|_{L^{2}\left(0, T ; \mathbb{H}^{2}(\Omega)\right)} \leq \alpha\|v\|_{L^{2}\left(0, T ; \mathbb{H}^{2}(\Omega)\right)}+\left\|F_{k}(x, t, 0,0)\right\|_{L^{2}\left(0, T ; \mathbb{L}^{2}(\Omega)\right)}+\left|\beta_{\varepsilon}(0)\right| d_{1} T
$$


It follows by $0<\alpha<1$ that

$$
\|v\|_{L^{2}\left(0, T ; \mathbb{H}^{2}(\Omega)\right)} \leq \frac{1}{1-\alpha}\left\|F_{k}(x, t, 0,0)\right\|_{L^{2}\left(0, T ; \mathbb{L}^{2}(\Omega)\right)}+\frac{1}{1-\alpha}\left|\beta_{\varepsilon}(0)\right| d_{1} T .
$$

Therefore, the proof is complete.

\section{The proof of the main result}

In this section, we prove that the solution to problem (5) converges to that of problem (1) when $\varepsilon \rightarrow 0$. From Lemma 3.3 and Lemma 3.5, we conclude that $u_{\varepsilon}$ in (7) exists a unique solution satisfying

$$
\begin{aligned}
& \left\|u_{\varepsilon}\right\|_{L^{2}\left(0, T ; \mathbb{H}_{0}^{1}(\Omega)\right)} \leq C, \\
& \left\|u_{\varepsilon}\right\|_{L^{2}\left(0, T ; \mathbb{H}_{0}^{2}(\Omega)\right)} \leq N\|F(\cdot, \cdot, 0,0)\|_{L^{2}\left(0, T ; \mathbb{L}^{2}(\Omega)\right)}+N\left|\beta_{\varepsilon}(0)\right| d_{1} T .
\end{aligned}
$$

Thus, there exists a subsequence of $\left\{u_{\varepsilon}\right\}$ still denoted by itself for convenience, and $u \in$ $L\left(0, T ; \mathbb{H}^{2}(\Omega)\right)$ such that

$$
\begin{aligned}
& u_{\varepsilon}^{k} \stackrel{\text { weak }}{\rightarrow} u^{k} \quad \text { in } L\left(0, T ; H^{2}(\Omega)\right), k=1,2, \ldots, d_{1}, \\
& u_{\varepsilon}^{k} \stackrel{\text { uniformly }}{\rightarrow} u^{k} \quad \text { in } C\left(\Omega_{T}\right), k=1,2, \ldots, d_{1} .
\end{aligned}
$$

Letting $\varepsilon \rightarrow 0$ in (5) and using the maximum principle, we arrive at

$$
\left\{\begin{array}{l}
\left(u^{k}\right)_{t}-L_{k} u-F_{k}(x, t, u, \nabla u) \geq 0, \\
u^{k}(x, 0)=u_{0}^{k}(x) \\
u^{k}(x, t)=g_{k}(x) .
\end{array}\right.
$$

Comparing (1) with (26), we only need to prove that

$$
\left[\left(u^{k}\right)_{t}-L_{k} u-F_{k}(x, t, u, \nabla u)\right]\left(u^{k}(x, t)-u_{0}^{k}(x)\right)=0
$$

holds. Note that $u^{k}(x, 0) \geq u_{0}^{k}(x)$ derived from Lemma 3.3. Thus, we can end the proof by showing

$$
\left(u^{k}\right)_{t}-L_{k} u-F_{k}(x, t, u, \nabla u)=0 \quad \text { when } u^{k}(x, t) \geq u_{0}^{k}(x) .
$$

In fact, there exists a positive constant such that for all $\left(x_{0}, t_{0}\right) \in\left\{u^{k}(x, t)>u_{0}^{k}(x)\right\}$,

$$
u_{\varepsilon}^{k}\left(x_{0}, t_{0}\right)>\alpha+u_{0}^{k}\left(x_{0}\right)
$$

holds when $\varepsilon$ is sufficiently small. Further, from (15) we have that

$$
0 \geq \beta_{\varepsilon}\left(u_{\varepsilon}^{k}-u_{0}^{k}\right) \geq \beta_{\varepsilon}(\alpha) \rightarrow 0 .
$$

Therefore, we conclude that

$$
\left(u^{k}\right)_{t}-L_{k} u-F_{k}(x, t, u, \nabla u)=0 \quad \text { in } L\left(0, T ; H^{2}(\Omega)\right) .
$$


Now, we prove the uniqueness by contradiction. Assume that $u_{1}=\left(u_{1}^{1}, u_{1}^{2}, \ldots, u_{1}^{d_{1}}\right)$ and $u_{2}=\left(u_{2}^{1}, u_{2}^{2}, \ldots, u_{2}^{d_{1}}\right)$ are the solutions of $(1)$ and $u_{1} \neq u_{2}$. That is, there exists at least one $k$ satisfying $u_{1}^{k} \neq u_{2}^{k}$. For simplicity, we assume that $\left\{u_{1}^{k}>u_{2}^{k}\right\}$ is not empty (if not, we assume $\left\{u_{1}^{k}<u_{2}^{k}\right\}$ is not empty), so that we have

$$
u_{1}^{k}>u_{2}^{k} \geq u_{0}^{k}(x) \quad \text { in }\left\{u_{1}^{k}>u_{2}^{k}\right\}
$$

This and (27) lead to

$$
\begin{aligned}
& \left(u_{1}^{k}\right)_{t}-L_{k} u_{1}-F_{k}\left(x, t, u_{1}, \Delta u_{1}\right)=0 \quad \text { in }\left\{u_{1}^{k}>u_{2}^{k}\right\}, \\
& \left(u_{2}^{k}\right)_{t}-L_{k} u_{2}-F_{k}\left(x, t, u_{2}, \Delta u_{2}\right) \geq 0 \quad \text { in }\left\{u_{1}^{k}>u_{2}^{k}\right\}, \\
& \left(u_{1}^{k}-u_{2}^{k}\right)_{t}-L_{k}\left(u_{1}-u_{2}\right)-\left(F_{k}\left(x, t, u_{1}, \Delta u_{1}\right)-F_{k}\left(x, t, u_{2}, \Delta u_{2}\right)\right) \leq 0 .
\end{aligned}
$$

By the maximum principle and (A8), we have that

$$
u_{1}^{k}-u_{2}^{k} \leq 0 \quad \text { in }\left\{u_{1}^{k}>u_{2}^{k}\right\}
$$

This is obviously contradictory. Therefore, we conclude that problem (1) has a unique solution. Moreover the estimate can be easily obtained by Lemma 3.5 and (28) with $\varepsilon \rightarrow 0$.

\section{Competing interests}

The authors declare that they have no competing interests.

\section{Authors' contributions}

YS and YS carried out the proof of the main part of this article, MW corrected the manuscript and participated in its design and coordination. All authors read and approved the final manuscript.

\section{Acknowledgements}

This work was supported by the National Nature Science Foundation of China (Grant No. 71171164) and the Doctorate Foundation of Northwestern Polytechnical University (Grant No. CX201235). The authors are sincerely grateful to the referees and the associate editor handling the paper for their valuable comments.

Received: 14 November 2012 Accepted: 3 December 2013 Published: 03 Jan 2014

\section{References}

1. Blanchet, $A$ : On the regularity of the free boundary in the parabolic obstacle problem application to American options. Nonlinear Anal. 65, 1362-1378 (2006)

2. Cox, J, Ross, S: The valuation of options for alternative stochastic processes. J. Financ. Econom. 3, 145-166 (1976)

3. Jeunesse, M, Jourdain, B: Regularity of the American Put option in the Black-Scholes model with general discrete dividends. Stoch. Process. Appl. 122, 3101-3125 (2012)

4. Kohler, M, Krzyzak, A: Pricing of American options in discrete time using least squares estimates with complexity penalties. J. Stat. Plan. Inference 142, 2289-2307 (2012)

5. Forsyth, PA, Vetzal, KR: Quadratic convergence for valuing American options using a penalty method. SIAM J. Sci. Comput. 23, 2095-2122 (2002)

6. Khaliq, AQM, Voss, DA, Kazmi, SH: A linearly implicit predictor-corrector scheme for pricing American options using a penalty method approach. J. Bank. Finance 30, 489-502 (2006)

7. Fasshauer, G, Khaliq, AQM, Voss, DA: Using meshfree approximation for multi asset American options. Mesh free methods. J. Chin. Inst. Eng. 27, 563-571 (2004)

8. Marcozzi, M: On the approximation of optimal stopping problems with application to financial mathematics. SIAM J. Sci. Comput. 22, 1865-1884 (2001)

9. Halluin, Y, Forsyth, PA, Labah, G: A penalty method for American options with jump-diffusion processes. Numer. Math. 97, 321-352 (2004)

10. Mariani, MC, SenGupta, I: Solutions to an integro-differential parabolic problem arising in the pricing of financial options in a Lévy market. Nonlinear Anal., Real World Appl. 12, 3103-3113 (2012)

11. Florescu, I, Mariani, MC: Solutions to an integro-differential parabolic problem arising in the pricing of financial options in a Lévy market. Electron. J. Differ. Equ. 62, 1-10 (2010)

12. SenGupta, I: Spectral analysis for a three-dimensional super radiance problem. J. Math. Anal. Appl. 375, 762-776 (2011) 
13. SenGupta, I: Differential operator related to the generalized super radiance integral equation. J. Math. Anal. Appl. 369 101-111 (2010)

14. Adams, RA: Sobolev Spaces. Academic Press, New York (1975)

15. Evans, LC: Partial Differential Equations, 2nd edn. Grad. Stud. Math., vol. 19. Am. Math. Soc., Providence (2010)

16. Folland, GB: Introduction to Partial Differential Equations, 2nd edn. Princeton University Press, Princeton (1995)

10.1186/1029-242X-2014-8

Cite this article as: Sun et al.: Second-order integro-differential parabolic variational inequalities arising from the valuation of American option. Journal of Inequalities and Applications 2014, 2014:8

Submit your manuscript to a SpringerOpen ${ }^{\circ}$ journal and benefit from:

- Convenient online submission

- Rigorous peer review

- Immediate publication on acceptance

Open access: articles freely available online

- High visibility within the field

- Retaining the copyright to your article

Submit your next manuscript at $>$ springeropen.com 